\section{$\underset{\substack{\text { hommes } \\ \text { \& migrations }}}{ }$}

\section{Hommes \& migrations}

Revue française de référence sur les dynamiques

migratoires

$1296 \mid 2012$

Le Mexique dans les migrations internationales

\title{
Le coyotage et les barrières imposées à la mobilité humaine par les états nationaux
}

Le cas des Mexicains et Centraméricains migrant vers les États-Unis

\section{David Spener}

Traducteur : Bernardo Toro

\section{(2) OpenEdition Journals}

\section{Édition électronique}

URL : http://journals.openedition.org/hommesmigrations/1504

DOI : 10.4000/hommesmigrations. 1504

ISSN : 2262-3353

Éditeur

Musée national de l'histoire de l'immigration

Édition imprimée

Date de publication : 1 mars 2012

Pagination : 46-53

ISSN : 1142-852X

Référence électronique

David Spener, "Le coyotage et les barrières imposées à la mobilité humaine par les états nationaux », Hommes \& migrations [En ligne], 1296 | 2012, mis en ligne le 01 mars 2014, consulté le 23 avril 2019. URL : http://journals.openedition.org/hommesmigrations/1504 ; DOI : 10.4000/ hommesmigrations. 1504 


\section{Le coyotage et les barrières imposées à la mobilité humaine par les États nationaux \\ Le cas des Mexicains et Centraméricains migrant vers les États-Unis}

Par David Spener, professeur, département de sociologie et d'anthropologie, université de Trinity (San Antonio, Texas)

Au Mexique et dans les pays centraméricains, les inégalités économiques poussent des milliers de personnes à abandonner leur pays d'origine

pour chercher une place sur le marché de l'emploi américain. N'ayant pour la plupart aucune chance d'entrer aux États-Unis par la voie légale, les migrants sont obligés de faire appel aux services de passeurs, les "coyotes". Soumis à toutes formes de violence, de rançonnage voire d'exécution par les cartels de la drogue ou les passeurs eux-mêmes, les migrants sont victimes de l'incurie de l'État mexicain. 
La dégradation des conditions de sécurité au Mexique durant la dernière décennie, surtout après l'arrivée de Felipe Calderón au pouvoir, en 2006, commence à se faire sentir. La guerre déclarée aux cartels de la drogue a eu comme conséquence involontaire l'accroissement désastreux et tragique des violations des droits de l'homme à l'endroit des migrants internationaux qui traversent le Mexique. Les enlèvements répétés et massifs de migrants sur les routes qui mènent à la frontière américaine et les massacres de migrants qui ont eu lieu dans l'État de Tamaulipas au cours des deux dernières années en constituent des exemples particulièrement frappants. Sans fournir de véritables preuves, on a prétendu que parmi les principaux responsables de ces faits de violence se trouvent les "coyotes" que les migrants morts ou enlevés avaient contactés.

Nous évoquerons dans cet article les dangers auxquels les migrants latino-américains doivent faire face lors de leur traversée du Mexique à partir de concepts et d'enseignements tirés d'un travail préalable ${ }^{(1)}$ portant sur la manière dont les migrants mexicains essayaient de traverser la frontière avec les États-Unis durant la période 1990-2005. J'aimerais mettre l'accent sur l'appui prêté par le gouvernement mexicain aux politiques d'"interdiction de la migration (2)" mises en place par le gouvernement américain, lesquelles ont rendu les migrants encore plus vulnérables à la violence issue de la guerre contre les trafiquants de drogue. Il me semble important de faire remarquer le rôle joué par les États, car les moyens de communication de masse insistent de manière souvent sensationnaliste sur la responsabilité des bandes organisées, en négligeant le rôle des politiques et des pratiques gouvernementales.

\section{Le "coyotage" comme réponse à l'interdiction du transit des migrants}

Comme nous le rappelle l'anthropologue Josiah Heyman, l'interdiction est devenue un élément central "des buts poursuivis par une frontière moderne". Par interdiction on entend ici les efforts déployés par les États "visant à arrêter le flux de biens et de personnes dans ou autour des frontières "(3)". Pendant un peu plus d'une décennie, et à la demande de son voisin du nord, l'État mexicain a intensifié ses efforts pour détecter et contrôler les flux des migrants irréguliers et des biens illicites vers les États-Unis. Ces efforts ne se sont pas seulement déployés aux portes d'entrée frontalières, dans les aéroports et les ports, mais aussi via les multiples barrages policiers, militaires et migratoires des principales autoroutes à l'intérieur du pays. En principe, ces efforts visaient à aider le gouvernement américain dans sa "guerre contre la drogue", ainsi qu’à réguler le flux de sans-papiers vers la frontière Sud des États-Unis. Ces migrants, qui affluent en 
nombre depuis plusieurs décennies, ne sont pas seulement mexicains, mais proviennent aussi du reste de l'Amérique latine. D'après certains observateurs, les mesures prises par l'État mexicain, en particulier sous le gouvernement de Vicente Fox au début des années 2000, avaient pour but de parvenir à un accord migratoire binational avec les États-Unis, mais cette tentative a échoué après les attentats terroristes du 11 septembre 2001.

L'installation à l'intérieur du Mexique d'un nombre important de barrages où l'on contrôle les documents migratoires ne manque pas d'ironie. Cette politique est due en grande partie aux pressions exercées par les États-Unis, alors même que dans ce pays de tels contrôles sont considérés comme arbitraires et illégaux. En effet, le quatrième amendement à la Constitution états-unienne (adoptée en 1787), l'un des dix premiers qui composent la Charte des droits, l'interdit.

L'un des principaux obstacles auxquels doivent faire face ceux qui, comme moi, cherchent une solution à la violence subie par les migrants latino-américains qui transitent par le Mexique, est la normalisation et la banalisation du contrôle du mouvement des étrangers effectué par les États, pratique qui est devenue totalement incontestable ${ }^{(4)}$. Lors de la conférence donnée par l'ancien président Vicente Fox à San Antonio, Texas, en 2007, un prêtre américano-mexicain l'a interrogé sur les mauvais traitements donnés aux Centraméricains qui traversent le Mexique pour se rendre aux États-Unis ; l'ironie contenue dans sa réponse a échappé au président mexicain : "Les instructions sont de respecter les droits de l'homme. Nous recevons 250000 Centraméricains au Mexique. Notre devoir est de les arrêter et de les renvoyer à leur pays d'origine. 250000! Sous la surveillance d'une Commission des droits de l'homme, nous avons construit des installations très modernes à Tapachula, Chiapas.

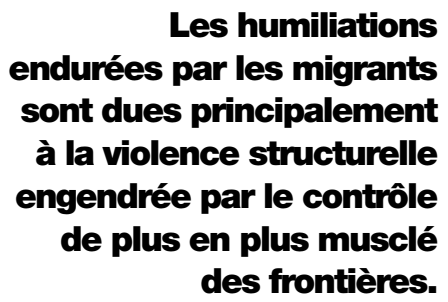

Quand nous arrêtons ces migrants centraméricains, nous leur offrons un toit, de la nourriture et un lit pendant le temps qu'ils restent chez nous (qui ne dépasse pas 24 ou 36 heures), après quoi nous les renvoyons chez eux $x^{(5)}$."

Dans d'autres travaux ${ }^{(6)}$, j'ai avancé l'idée que l'organisation (actuelle et passée) instaurée à la frontière entre les États-Unis et le Mexique ressemble à un système d'apartheid global ${ }^{(7)}$. Il favorise la ségrégation de la population en termes raciaux et économiques et la surexploitation des migrants mexicains qui ont réussi à contourner l'interdiction d'entrer sur le territoire états-unien et bénéficient de conditions de travail inférieures à celle de la population états-unienne. En ce sens, l'interdiction représente une sorte de violence structurelle, dans laquelle il n'y a pas d'agresseur 
individuel qui commet un acte isolé. L'organisation de la société est telle que "la violence est incorporée dans la structure sociale et se manifeste comme pouvoir inégal et par voie de conséquence comme facteur de vie inégale ${ }^{(8)}$."

Pendant un siècle environ, l'une des principales stratégies des Mexicains pour entrer illégalement dans le territoire américain a été d'engager des passeurs connus familièrement sous le nom de "coyotes". Cette stratégie que j’ai appelée le "coyotage" réunit un "ensemble de pratiques mises en place par les 'coyotes' pour traverser les frontières internationales à la demande des migrants, des amis et des parents des migrants ou de leurs employeurs américains ${ }^{(9)}$ ". Il n'est pas surprenant que les migrants subalternes d'Amérique centrale, lesquels sont souvent davantage dans le besoin que les Mexicains, aient fait du "coyotage" l'une des principales stratégies pour surmonter les barrières qu'on leur impose avec de plus en plus de rigueur au Mexique.

\section{Le "coyotage", le trafic de drogues, I’État et la violence contre les migrants au Mexique}

Pendant la période où je m'occupais des traversées illégales de la frontière texane réalisées par les Mexicains dans les années quatre-vingt-dix et deux mille, les médias de masse répercutaient mécaniquement les déclarations du gouvernement américain qui désignait les "coyotes" comme la cause directe de la souffrance et de la mort des migrants ${ }^{(10)}$. Avec d'autres analystes, je suis toutefois arrivé à la conclusion que la source principale de leurs souffrances était la volonté américaine de rendre de plus en plus difficile la traversée de la frontière en mettant en place l'opération Blocage en $1993^{(11)}$. Les humiliations endurées par les migrants sont dues principalement à la violence structurelle engendrée par le contrôle de plus en plus musclé des frontières. Vers la fin de mon étude de terrain, au milieu des années deux mille, quand les conditions de sécurité au Tamaulipas, État qui jouxte le Texas, ont commencé à se dégrader à cause du conflit dans le milieu de la drogue entre le cartel du Golfe et son bras armé, les Zetas, on a parlé d'une implication croissante des trafiquants de drogue dans la circulation des migrants à travers les villes frontalières. On ne savait pas encore si le rôle des cartels se limitait à l'encaissement du droit du sol exigé aux coyotes qui opéraient dans leurs territoires ou bien s'ils participaient directement au "coyotage". Dans une étude ultérieure sur l'activité des “coyotes", Izcara Palacios explique que les coyotes du Tamaulipas ont gardé leur autonomie, du moins sur le plan opérationnel, malgré le pouvoir croissant des cartels de la drogue ${ }^{(12)}$. Les “coyotes" et les cartels ne se seraient donc pas rassemblés en une seule entité plus violente et sinistre encore. 


\section{Différences entre les expériences des Mexicains et des Centraméricains en transit au Mexique}

Dans les entretiens que j'ai pu réaliser, les "coyotes" et les migrants mexicains ont reconnu que les Centraméricains déployaient bien plus d'efforts que les Mexicains pour rejoindre les États-Unis. Les religieuses qui administraient la Maison du migrant à Reynosa, dans l'État de Tamaulipas, ont expliqué que l'expérience des Centraméricains dans leur passage par le Mexique était un véritable "calvaire". En premier lieu, ils devaient traverser, dans le meilleur des cas, une frontière de plus que les Mexicains, et donc échapper au moins deux fois aux mêmes menaces. En deuxième lieu, les Centraméricains devaient éviter les barrages douaniers et d'immigration dans les régions frontalières et subir de nombreuses fouilles sur les autoroutes qui mènent au nord du pays. De ce fait, beaucoup de Centraméricains préféraient prendre des trains de marchandises pour se rendre au Nord, avec tous les risques que ce type de transport implique. D'autres étaient obligés d'engager des "coyotes" qui leur servaient non seulement de guide, mais qui les conduisaient tout au long de leur périple au Mexique et les hébergeaient dans les régions frontalières. Cela engendrait des dépenses bien supérieures à celles engagées par les migrants mexicains.

Plus important encore, ce voyage clandestin à travers le Mexique les exposait au risque d'être attaqués, kidnappés, rackettés et même assassinés, surtout après l'arrivée au pouvoir de Felipe Calderón, en 2006, quand une vague de violence, sans précédent depuis la révolution, s'est déchaînée sur le pays. Même si cela paraît contradictoire étant donné leur dénuement et leur désespoir, les migrants centraméricains représentaient une affaire particulièrement juteuse pour les bandes du crime organisé. Comme les migrants mexicains, les Centraméricains s'étaient mis d'accord avec leurs familles aux États-Unis pour ne payer aux "coyotes" la totalité de la somme qu'une fois arrivés à destination. Ces migrants représentaient donc une source possible d'extorsion de plusieurs milliers de dollars pour ceux qui savaient qu'ils se rendaient aux États-Unis. Il n'est donc pas surprenant que l'enlèvement des migrants en transit au Mexique ait atteint des niveaux aussi alarmants ces dernières années. La Commission nationale de droits de l'homme a estimé qu'en 201011333 migrants avaient été enlevés au cours des 214 incidents enregistrés ${ }^{(13)}$.

\section{Tamaulipas, terre de tragédies}

Les nombreux massacres qui ont eu lieu dans le secteur de San Fernando, au Tamaulipas, en 2010 et 2011, ont démontré que la vulnérabilité des droits de l'homme au Mexique avait atteint, avec les expériences des migrants, le niveau d'une véritable crise nationale. On pensait que les "coyotes" qui avaient organisé la traversée avaient participé directement à ces faits barbares. Cette participation n'a toutefois pas été 
démontrée. La seule certitude est que les criminels faisaient partie des Zetas, le cartel du trafic de la drogue. On peut envisager plusieurs hypothèses pour expliquer la participation des "coyotes" dans ces faits de violence et définir les stratégies à suivre pour freiner les abus dont sont victimes les migrants centraméricains. Je présente ici six hypothèses qui ne s'excluent pas forcément : 1. Les Zetas ont la mainmise sur la circulation des migrants sans papiers centraméricains à travers le Mexique. Si cette hypothèse s'avère juste, il n'y a plus de "coyotes" indépendants. Les migrants n'ont donc pas le choix, ils sont obligés d'accepter le transport et l'hébergement proposés par les membres de cette bande, l'une des plus redoutables de la planète. 2. Les Zetas ne contrôlent pas le transport des migrants à travers le Mexique, mais se font passer pour des "coyotes" à la frontière afin que les migrants transportent leur drogue. Les "coyotes" qui accompagnent les migrants dans leur traversée du Mexique laissent ceux-ci aux mains des trafiquants une fois qu'ils ont atteint la frontière nord. 3. Les Zetas ne s'occupent pas du déplacement des migrants à travers le territoire

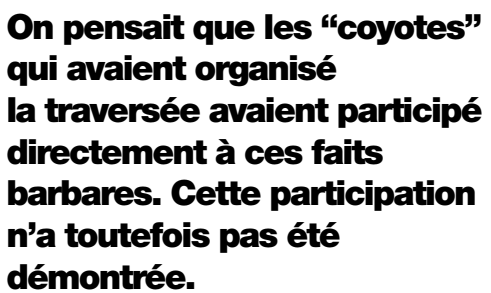
mexicain, ils se contentent de faire payer un droit du sol aux "coyotes", lesquels continuent de transporter et de guider les migrants dans leur périple mexicain. Le "coyotage" serait ainsi une affaire comme n'importe quelle autre victime du racket des bandes organisées. Les dommages subis par les migrants seraient dus au fait que leurs "coyotes" n'ont pas payé leur tribut aux Zetas. 4. Les Zetas ne s'occupent pas du "coyotage", mais surveillent le déplacement des migrants à travers le territoire mexicain dans le but d'extorquer de l'argent aux familles des migrants qui se trouvent aux États-Unis. Les Zetas imiteraient les stratégies de certaines bandes spécialisées dans l'enlèvement de migrants sans papiers qui agissent dans les États de Californie, de l'Arizona et du Texas. 5. Les "coyotes" et les Zetas ne forment pas une même bande, mais ils se rendent des services. Les "coyotes" abandonnent certains migrants aux Zetas qui les volent, rackettent, réduisent à l'esclavage et à la prostitution, etc. Les Zetas sont, en effet, une bande plus importante, mieux organisée, fortement armée et prête à punir les "coyotes" qui n'accèdent pas à leurs demandes. 6. Les "coyotes" et les Zetas comptent sur l'appui des mêmes agents gouvernementaux pour se déplacer librement dans le territoire mexicain. Ces agents constituent un élément indispensable du lieu où opèrent ces bandes, il s'agit de policiers de tous les niveaux, des militaires et du personnel de l'Institut national de la migration ${ }^{(14)}$. Les migrants qui ont été enlevés et pris en otage au Tamaulipas ont déclaré que c'étaient les inspecteurs de l'Institut national de la migration eux-mêmes qui les avaient livrés à leurs ravisseurs ${ }^{(15)}$. 


\section{La nouvelle loi migratoire au Mexique}

Devant la violation systématique des droits de l'homme à l'encontre des migrants décrite plus haut, l'État mexicain a promulgué une nouvelle loi migratoire en mai 2011. Son objectif était de mettre un terme aux atteintes aux droits de l'homme des migrants internationaux qui transitent par le Mexique. Plus spécifiquement, la nouvelle loi autorise la migration irrégulière à l'intérieur du territoire mexicain, sanctionne les autorités qui violent les droits de l'homme des migrants et propose un nouveau type de visa qui permet d'entrer temporairement au Mexique et de voyager dans tout le pays pendant 180 jours $^{(16)}$.

L'octroi d'un visa aux migrants centraméricains qui jusqu'à présent s'étaient vus obligés d'entrer dans les pays de manière illégale devrait amoindrir les risques que ceux-ci encourent dans leur périple vers les États-Unis, si toutefois les migrants pauvres remplissent les conditions requises pour l'obtention desdits visas. Les défenseurs des droits de l'homme se demandent si l'octroi des visas sera suffisamment rapide et à l'abri de la corruption pour réduire la vulnérabilité des migrants. Les modalités pratiques de cette nouvelle loi n'ont, en effet, pas encore été déterminées. Certains hommes politiques et journalistes ont même proposé que l'on élimine l'exigence d'un visa aux migrants qui partent pour les États-Unis et que l'on supprime l'Institut national de la migration ${ }^{(17)}$.

Même si la nouvelle loi migratoire réussit à mettre en place un système d'obtention de visas efficace, à l'abri de la corruption et accessible pour ceux qui en ont besoin, les migrants pourraient continuer à faire face à des dangers tels que les enlèvements. Ainsi qu'on l'a souvent constaté, l'un des principaux risques qui guettent les migrants est d'être repérés comme tels lors des très nombreux contrôles auxquels ils sont soumis sur les autoroutes qui mènent à la frontière américaine. Si les Zetas continuent d'acheter les services des policiers, des militaires et des agents de l'Institut national de la migration qui contrôlent régulièrement les papiers des passagers d'autobus et des voitures, il sera toujours facile d'identifier des migrants centraméricains, même s'ils ont un visa. Il est fort probable que les bandes organisées continuent à engager des employés publics comme indicateurs. Si son but est vraiment d'en finir avec les enlèvements, il est indispensable que le gouvernement mexicain ne se limite pas à octroyer des visas aux migrants et qu'il supprime les barrages à l'intérieur du pays. Ce n'est pas seulement leur qualité de sans-papiers qui rend les migrants vulnérables, mais aussi les multiples contrôles auxquels ils sont soumis par les autorités policières, militaires et migratoires. Ainsi qu'on l'a souvent démontré, ces contrôles n'ont pas réussi à freiner le trafic de stupéfiants et d'armes dans le pays, on peut donc difficilement prétendre qu'il faut les maintenir pour préserver la sécurité publique. 


\section{Notes}

1. David Spener, Clandestine Crossings: Migrants and Coyotes on the Texas-Mexico Border, Ithaca, New York, Cornell University Press, 2009.

2. Dans mon livre Clandestine Crossings, j'explique comment les pratiques d'interdiction de la migration du gouvernement états-unien constituent un exemple du système d'“apartheid global".

3. Josiah McC. Heyman, "Why interdiction ? Immigration control at the United States-Mexican border", in Regional Studies, vol. 33, n 7, 1999, pp. 619-620.

4. Joseph Nevins, "Thinking out of bounds: A critical analysis of academic and human rights writings on migrant deaths in the U.S.-Mexico border region", in Migraciones Internacionales, vol. 2, n 2, 2003, pp. 171-90; Joseph Nevins, "A beating worse than death: Imagining and contesting violence on the U.S.-Mexico borderlands", in Ameriquests, vol. 2 , $\mathrm{n}^{\circ}$ 2, 2005. http://ejournals.library. vanderbilt.edu/ameriquests/index.php (téléchargé le 25 août 2006).

5. 27 mars 2007 au Laurie Auditorium, Trinity University, San Antonio, Texas.

6. David Spener, Clandestine Crossings: Migrants and Coyotes on the Texas-Mexico Border, op. cit.; David Spener, "El apartheid global, el coyotaje y el discurso de la migración clandestina: Distinciones entre violencia personal, estructural y cultural", in Migración y Desarrollo, n 10, 2008, pp. 127-156.

7. Gernot Kohler, "Global Apartheid", World Order Models Project, Working paper n 7, New York, Institute for World Order, 1978 ; Gernot Kohler, "The three meanings of global apartheid: Empirical, normative, and existential", in Alternatives, $\mathrm{n}^{\circ}$ 20, 1995, pp. 403-413.

8. Johan Galtung, "Violence, peace, and peace research", in Journal of Peace Research, vol. 6, n 3, 1969, pp. 170-171.

9. David Spener, Clandestine Crossings: Migrants and Coyotes on the Texas-Mexico Border, op. cit., p. 95.

10. Johan Galtung, "Violence, peace, and peace research", op. cit.

11. David Spener, Clandestine Crossings: Migrants and Coyotes on the Texas-Mexico Border, op. cit.; Andreas Feldman, et Jorge Durand, "Mortandad en la frontera", in Migración y Desarrollo, n 10, 2008, pp. 11-36 ; Jezmin Fuentes, Henry L'Esperance, Raúl Pérez and Caitlin White, "Impacts of U.S. immigration policies on migration behavior", in Wayne A. Cornelius and Jessa M. Lewis (dir.), Impacts of Border Enforcement on Mexican Migration: The View from Sending Communities, Boulder, Lynne Rienner, 2007, pp. 53-73 ; Jacqueline Hagan, Migration Miracle: Faith, Hope, and Meaning on the Undocumented Journey, Cambridge, Massachusetts, Harvard University Press, 2008.

12. Simón-Pedro Izcara Palacios, "La adicción a la mano de obra ilegal: Jornaleros tamaulipecos en Estados Unidos", in Latin American Research Review, vol. 45, n 1, 2010, pp. 55-75 ; Simón-Pedro Izcara Palacios, "Militarización de la frontera e inmigración ilegal: los jornaleros tamaulipecos", in Estudios Fronterizos, vol. 10, n 20, 2009, pp. 9-45.

13. Nadia Sanders, " 11,333 migrantes fueron secuestrados en México en 2010: CNDH", in CNN.com, 22 février 2011. http://mexico.cnn.com/nacional/2011/02/22/11333-migrantes-fueron-secuestrados-en-mexico-en-2010-cndh (téléchargé le 15 septembre 2011).

14. LINM (Institut national de la migration) est une dépendance du gouvernement mexicain dédiée à l'interdiction de séjour, à la détention et à la déportation des migrants sans papiers au Mexique, y compris ceux qui transitent par le territoire pour se rendre aux États-Unis (http://www.inm.gob.mx/). Voir Howard Campbell, Drug War Zone: Frontline Dispatches from the Streets of El Paso and Juárez, Austin, Texas, University of Texas Press, 2009.

15. Víctor Ballinas, "Colusión del INM en secuestros 'no es nueva'", in La Jornada, 11 mai 2011, p. 8 ; "Agentes migratorios mexicanos cobraban \$430 por “vender' migrantes”, in El Universal, 10 mai 2011; Alfredo Méndez, "Denuncian migrantes que agentes del INM los vendieron a los Zetas", in La Jornada, 10 mai 2011, p. 7 ; "Señalan a INM por plagios de migrantes", in Reforma, 9 mai 2011.

16. “¿Qué dice la nueva Ley de Migración?”, in El Universal, 24 mai 2011 ; Fabiola Martínez, "Dará el INM opciones de visa a migrantes de Centroamérica", in La Jornada, 15 août 2011, p. 17 ; Organisation internationale pour les migrations, "Promulgación Nueva Ley de Migración en México", in Boletín ONU, Comunicado n 11/129, 25 mai 2011. http://www.cinu.mx/comunicados/2011/05/promulgacion-nueva-ley-de-migr/ (téléchargé le 15 septembre 2011); Secrétariat du gouvernement du Mexique, "Promulga Calderón la Ley de Migración", México, Comunicado de prensa, 24 mai 2011. http://www.inm.gob.mx/index.php /page/Notadeldia_240511 (téléchargé le 15 septembre 2011).

17. Agence Reforma, "Piden a INM abrir reglamento de ley", in El Golfo, 31 août 2011 ; "Recibe Segob propuestas para reglamento a Ley de Migración", in Milenio Online, 13 septembre 2011.

www.milenio.com/cdb/doc/noticias2011/ 75d9bcfb7cba54dfc1973e6f47b90da0 (téléchargé le 15 septembre 2011); Andrés Timoteo Morales, "Propone Solalinde desparecer Instituto Nacional de Migración”, in La Jornada,

2 juillet 2011, p. 7 ; Pedro Miguel, "Cuestión de visas”, in La Jornada, 6 septembre 2011. 